\title{
Astrophysical lasers operating in optical Fe II lines in stellar ejecta of $\eta$ Carinae
}

\author{
S. Johansson ${ }^{1}$ and V. S. Letokhov ${ }^{2,1}$ \\ ${ }^{1}$ Lund Observatory, Lund University, PO Box 43, 22100 Lund, Sweden \\ e-mail: [sveneric.johansson;Vladilen.Letokhov]@astro.lu.se \\ ${ }^{2}$ Institute of Spectroscopy, Russian Academy of Sciences, Troitsk, Moscow region 142190, Russia
}

Received 3 March 2004 / Accepted 25 August 2004

\begin{abstract}
After the discovery of space masers based on $\mathrm{OH}$ radicals (Weaver et al. 1965) and $\mathrm{H}_{2} \mathrm{O}$ (Cheung et al. 1969) such microwave lasers have been found to work in more than 100 molecular species (Elitzur 1992; Townes 1997), as well as in highly excited $\mathrm{H}$ atoms (Strelnitski et al. 1996). In the IR region $(10 \mu \mathrm{m})$, the effect of stimulated emission of radiation in the $\mathrm{CO}_{2}$ molecule has been discovered in the Martian and Venus' atmospheres (Betz et al. 1976; Mumma et al. 1981). We report here on the discovery of laser action in the range $0.9-2 \mu \mathrm{m}$ in several spectral lines of Fe II, which are associated with transitions from "pseudo-metastable" states populated by spontaneous transitions from Ly $\alpha$ pumped Fe II levels. The intense Ly $\alpha$ radiation is formed in the HII region of gas condensations close to the star $\eta$ Car. The laser transitions form together with spontaneous transitions closed radiative cycles, one of which includes the extremely bright 2507/09 A lines. Closed radiative cycles, together with an accidental mixing of energy levels, may provide an explanation of the abnormal intensities of these UV non-lasing lines. Using the complicated energy level diagram of Fe II we present those peculiar features, which are essential for the inverted population and laser effect: the pumping, the level mixing, and the "bottle neck" for spontaneous decay. The laser action is a new indicator of non-equilibrium and spatially non-homogeneous physical conditions as well as a high brightness temperature of $\operatorname{Ly} \alpha$ in ejecta from eruptive stars. Such conditions are very difficult to probe by existing methods, and we propose some future experiments. The fact, that the lasing near-IR lines appear in the spectrum with about the same intensity as non-lasing lines is discussed and compared with the situation in masers.
\end{abstract}

Key words. atomic processes - line: formation - radiation mechanisms: non-thermal - stars: individual: $\eta$ Carinae

\section{Introduction}

A gas condensation formed by expelled material in the neighborhood of a bright star is an optimal site for hydrogen atoms to be photoionized by extreme ultraviolet (EUV) radiation under high-vacuum conditions. The central star emits the EUV radiation, and hydrogen is ionized by photons with $\lambda<912 \AA$. Collisions with electrons at a low rate yield the characteristic recombination spectrum of atomic hydrogen. The intense Ly $\alpha$ line at $1215 \AA$, which cannot be observed from Earth because of interstellar absorption, contributes about $70 \%$ of the energy radiated by the recombination lines. However, Ly $\alpha$ radiation is observed indirectly through its interaction with atoms and ions of other elements, particularly through resonant photoexcitation due to wavelength coincidences. Such an accidental coincidence of spectral lines (the Bowen (1935) mechanism) is a rare phenomenon. Since the spectral lines of free atoms and ions are very narrow the Bowen mechanism has no practical use in present-day lasers. However, this selective excitation mechanism generates some spectral lines in astrophysical plasmas, which contain a mixture of atoms and ions exposed to intense spectral lines of hydrogen, helium and other abundant light elements. The possibility of laser action in stellar atmospheres based on processes such as the Bowen mechanism was considered fairly long ago (Letokhov 1972; Lavrinovich \& Letokhov 1974), but the resulting line emission has been difficult to resolve from the stellar background radiation.

The observational conditions changed radically with the launch of the Hubble Space Telescope (HST) and the High Resolution Spectrograph (HRS). Its successor, the Space Telescope Imaging Spectrograph (STIS), provides high spectral resolution in a broad wavelength range (1150-10400 $\AA$ ) and a near-diffraction-limited angular resolution $(\sim 0.1$ arcsec $)$ (Kimble et al. 1998). Beginning in 1998, the eruptive star $\eta$ Car became one of the most remarkable targets for this instrument (Gull et al. 1999). It is an exceptionally interesting astrophysical object expelling enormous amounts of material into its surroundings. In the immediate vicinity of the central star at a distance of 100-1000 stellar radii $\left(r_{\mathrm{s}}=3 \times 10^{13} \mathrm{~cm}\right)$, compact gas condensations (GC), called the Weigelt blobs B, $\mathrm{C}$ and $\mathrm{D}$, of exceptional brightness have been discovered 


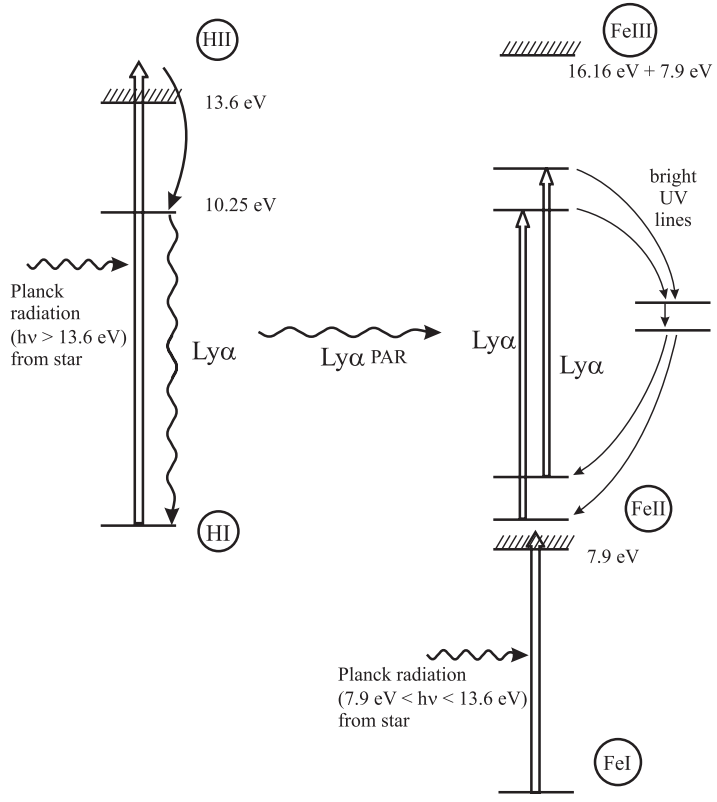

Fig. 1. Illustration of the main radiative processes in the blobs outside $\eta$ Car, involving photoionization of $\mathrm{HI}$ and $\mathrm{Fe}$ I by stellar blackbody radiation and subsequent resonant excitation of Fe II by intense $\mathrm{H}$ Ly $\alpha$ radiation.

(Weigelt \& Ebersberger 1986). They are unique astrophysical plasmas having a higher hydrogen density $\left(N_{\mathrm{H}} \approx\right.$ $10^{7}-10^{10} \mathrm{~cm}^{-3}$ ) (Davidson \& Humphreys 1997) than planetary nebulae $\left(N_{\mathrm{H}} \approx 10^{4} \mathrm{~cm}^{-3}\right)$ and located only a few light days away from the central star.

For clarity we illustrate in Fig. 1 the sequence of basic radiative processes in the blobs, initiated by stellar blackbody (Planck) radiation acting on $\mathrm{H} \mathrm{I}$ and Fe I and followed by pumping due to an accidental resonance (PAR) between FeII and Ly $\alpha$. A modelling of the spectrum of the Weigelt blobs (Verner et al. 2002) has verified the importance of $\operatorname{Ly} \alpha$ pumping to reproduce the fluorescent Fe II lines and continuum pumping to reproduce low-excitation Fe II as well as [Fe II] lines. As $N_{\mathrm{H}}$ is relatively high, the blob will almost completely absorb the Lyman-continuum radiation from the central star. The Strömgren border, separating the regions of completely ionized (HII region) and neutral hydrogen (HI region), is thus located inside the blob. The HII region is adjacent to the HI region. The intensity of the Ly $\alpha$ recombination line in the HII region of the blob is $10^{3}-10^{4}$ times higher than that coming from the central star (Klimov et al. 2002; Johansson \& Letokhov 2001b). We get a unique situation in the HI region where a partially ionized mixture of many elements is exposed to an intense Ly $\alpha$ flux coming from the HII region. This is similar to a typical "laser" situation where the pumping flash lamp irradiates the nearby active laser medium.

Preliminary data on laser effects in the Weigelt blobs have been published in the form of short letters (Johansson \& Letokhov 2002, 2003). The present paper contains a more detailed treatment of the laser schemes related to the energy level diagram of Fe II, a detailed analysis of the amplification in various schemes and a discussion of the spectral width of laser lines. Below we give a brief outline of the paper.
The iron atoms occur in the $\mathrm{HI}$ region at a density of about $N_{\mathrm{Fe}} \approx 10^{-4} \times N_{\mathrm{H}}$, and they are easily photo-ionized by the stellar photospheric radiation in the spectral window $7.9 \mathrm{eV}<$ $h v<13.6 \mathrm{eV}$ (Fig. 1). The $\mathrm{Fe}^{+}$ion has a line-rich spectrum, and some lines coincide in wavelength with the wide Ly $\alpha$ line resulting in a selective photo-excitation to high energy levels (Brown et al. 1979; Johansson \& Jordan 1984). This is discussed in Sect. 2 as well as a general scheme of the radiative decay routes of the Ly $\alpha$-excited Fe II levels. These radiative pathways are numerous and peculiar because of the complex Fe II energy level diagram, which offers both accidental level mixing (ALM) and the presence of "pseudo-metastable" $(P M)$ high-lying states (Sect. 3). The latter have lifetimes of about $1 \mu \mathrm{s}-1 \mathrm{~ms}$ and they create "bottle-necks" in the chain of spontaneous radiative decays of the Ly $\alpha$-excited Fe II levels.

In Sect. 4 the formation of population inversion in the PM states of Fe II, a basic requirement for lasing effect, is described as well as the size of the amplification coefficient. Two types of "bottle-neck", occurring in PM states with ms and $\mu$ s lifetimes, respectively, are discussed separately in great detail in Sects. 5 and 6. The ms-"bottle-neck" (Sect. 5) provides a) a strong radiative cycle in Fe II generated by Ly $\alpha$ pumping, b) lasing in spectral lines at $1.68 \mu \mathrm{m}$ and $1.74 \mu \mathrm{m}$, and c) the basis for the explanation of the anomalously bright non-lasing UV lines at $2507 / 09 \AA$. The $\mu$ s "bottle-neck" (Sect. 6) provides laser effect in several lines in the range $0.9-1.0 \mu \mathrm{m}$, observed in HST/STIS spectra of the Weigelt blobs. For completeness, we are also considering (Sect. 7) the combination of strong and weak radiative cycles, where a ms-"bottle-neck" creates strong lasing lines at $\lambda \lambda 9617 / 9913$. For the $\lambda \lambda$ 9617/9391 lines we also observe a peculiar intensity ratio, which is in disagreement with the predicted branching ratio. In Sect. 8 we briefly discuss potential sources of $\operatorname{Ly} \alpha$ radiation providing the pumping of the $\mathrm{Fe}^{+}$ions in the Weigelt blobs.

Sections 9 and 10 contain a qualitative discussion of the differences between astrophysical lasers and masers from an observational point of view. The evidence for astrophysical masers is mostly based on their high brightness and narrow line widths, observed by high-resolution radio telescopes. However, the astrophysical laser lines may not be brighter than ordinary lines, and the high spectral resolution $\left(\simeq 10^{5}\right)$ of optical telescopes/spectrometers (e.g. HST/STIS) is too small to observe their narrow Doppler profiles. The possibility of using laser heterodyne Brown-Twiss-Townes interferometry is proposed in Sect. 10.

\section{Radiative excitation and relaxation of Fe II levels populated by absorbed Ly $\alpha$ radiation}

A large number of observed as well as predicted absorption lines of Fe II fall within a spectral width of $3 \AA$ around Ly $\alpha$. The transitions start from low metastable states as illustrated in Fig. 2 (see Johansson \& Jordan 1984). There are about 15 emission lines/Å observed in the laboratory spectrum of Fe II in the wavelength region around $\operatorname{Ly} \alpha$. The number is probably even higher for absorption lines, some of which could be pumped by $\operatorname{Ly} \alpha$ in astrophysical plasmas. Consequently, numerous Ly $\alpha$ pumped fluorescence lines of Fe II have been 


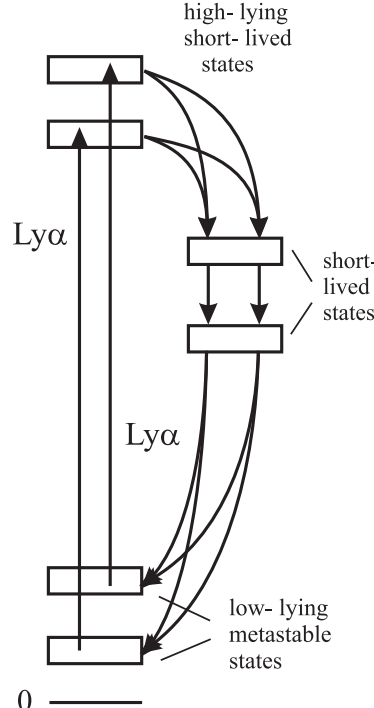

a

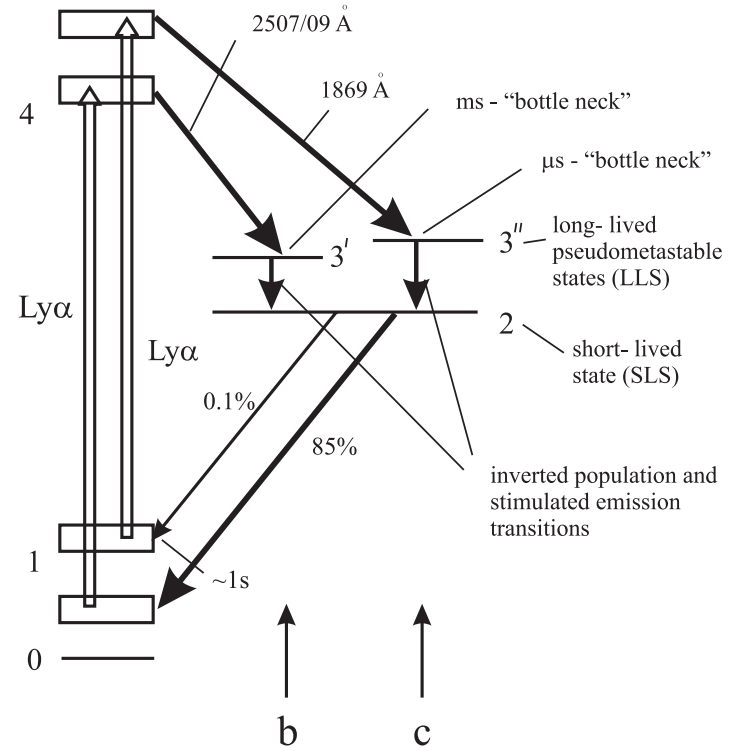

Fig. 2. Three types of radiative decay schemes of high-lying Fe II levels photoexcited by Ly $\alpha$ : a) a fast radiative decay down to metastable states via short-lived levels without any "bottle neck" effect; b) a radiative decay via high, pseudo-metastable states (the "bottle neck" effect) with formation of an inverted population; c) a radiative decay with "bottle neck" effect, inversion of population and return to the initial state.

identified in spectra of various emission line sources, eg. chromospheres of the sun and cool stars (Jordan 1988a,b; Harper et al. 2001), symbiotic stars (Hartman \& Johansson 2000), and the environment of AGNs (Netzer 1988).

The Weigelt blobs outside $\eta$ Car are rather special because of their high optical density for $\operatorname{Ly} \alpha, \tau_{\alpha} \approx 10^{8}$, and their short distance to the central star. The ratio of the Einstein coefficients for Ly $\alpha$ and the pumped absorption lines of $\mathrm{Fe}$ II is $\approx 10^{2}$, and since all $\mathrm{Fe}$ atoms in the $\mathrm{HI}$ region are ionized the abundance ratio $N_{\mathrm{Fe}^{+}} / N_{\mathrm{H}} \approx 10^{-4}$. Since the optical density for the Fe II absorption lines is in the range $\tau_{\text {FeII }} \approx 10-100$, the resonant intervals within the wide $\operatorname{Ly} \alpha$ profile are fully absorbed. A significant density of absorption lines should result in a total absorption of Ly $\alpha$ in the H I region of the blob.

The photoselective excitation rate of state 4 is defined by

$W_{\mathrm{exc}}^{14}=A_{41} \frac{g_{4}}{g_{1}}\left[\exp \left(\frac{h v_{14}}{k T_{\alpha}}\right)-1\right]^{-1}$,

where the indices 1 and 4 correspond to the level notations in Figs. 2b,c. The Einstein coefficient for spontaneous decay is $A_{41}=1.2 \times 10^{7} \mathrm{~s}^{-1}$ (Kurucz 2003), and $T_{\alpha}=T(\operatorname{Ly} \alpha)$ is the brightness (or effective) temperature of Ly $\alpha$ inside blob B. According to the qualitative picture given above the stellar Lyman continuum radiation is transformed into intense Ly $\alpha$ radiation. A more detailed analysis of this "spectral compression" effect is presented in (Johansson \& Letokhov 2004), and it shows that $T_{\alpha}$ is comparable with the effective temperature of the stellar photosphere. In the case of $\eta$ Car, we adopt $k T_{\alpha} \approx 1.0-1.5 \mathrm{eV}\left(T_{\alpha} \approx(12-18) \times 10^{3} \mathrm{~K}\right)$, which means a photoselective excitation rate of $W_{\mathrm{exc}}^{14} \approx 10^{3}-10^{4} \mathrm{~s}^{-1}$. Let us emphasize that without compensation for the dilution factor by the "spectral compression" effect the value of $W_{\text {exc }}^{14}$ will be about $1 \mathrm{~s}^{-1}$, making it impossible to provide an inverted population in the $3 \rightarrow 2$ transition.
The numerous possible pathways of the radiative relaxation (decay) of the Ly $\alpha$-excited Fe II levels can be grouped in two categories as illustrated in Fig. 2:

- a spontaneous decay back to the low metastable states without any accumulation in higher states (Fig. 2a);

- spontaneous decay via long-lived higher states ("bottle neck"), which cause an inverted population with an associated stimulated emission (Figs. 2b,c).

The first type of pathway (Fig. 2a) is dominating for all photoexcited states, and the type illustrated in Fig. 2b occurs as a result of $A L M$, discussed in Sect. 2. The general case is a combination of (1) and (2), where the branching fractions from level 4 determine the population in 3 . The branching fractions for the decay feeding the long-lived states are thus determined by the strength of the level mixing. In this paper we focus on case (2), i.e. those Ly $\alpha$ pumped Fe II levels having large branching fractions to feed the $P M$ states. It should be pointed out that the continuum pumping, shown by Verner et al. (2002) to increase the population of the low metastable states through cascading, cannot directly populate the PM states due to parity considerations.

If level 2 in case (2) decays to the initial state, i.e. the level being pumped, we obtain a closed radiative cycle (Johansson $\&$ Letokhov 2003). This is determined by the possible decay channels of state 2 and their branching fractions, which may vary among the set of short-lived states (lifetime of a few ns) fed by the decay from the $P M$ states. The distribution of the decay of state 2 is also important for permanent pumping of the low-lying metastable states, with lifetimes of the order of one second. Two examples of pathways containing radiative "bottle necks", according to the scheme in Figs. 2b,c, are discussed in detail in Sects 5-6 and a combination of them in Sect. 7. They represent closed radiative cycles, but with different 


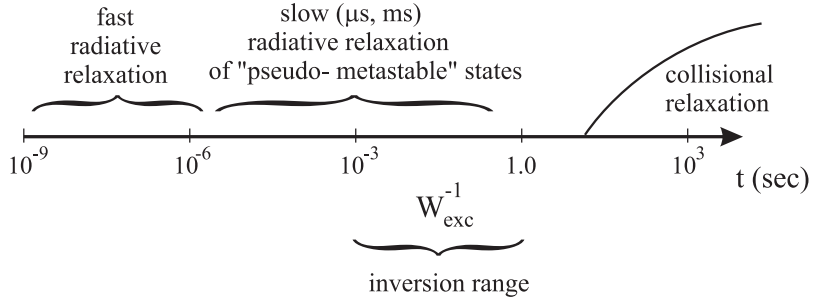

Fig. 3. Time scale of spontaneous radiative and collisional relaxation of highly excited Fe II states. An intermediate range including pseudometastable states is favourable obtaining an inverted population in the $3 \rightarrow 2$ transition.

efficiencies, as the branching fractions differ considerably in those transitions, that bring them back to the initial level. These two pathways of radiative decay have also different lifetimes of the "bottle neck" levels $3^{\prime}$ and $3^{\prime \prime}$ : a ms-lifetime for level 3' and a $\mu$ s-lifetime for $3^{\prime \prime}$ (Figs. 2b,c). The branching fractions for the weakly closed cycle $(0.1 \%)$ and for the strongly closed cycle $(85 \%)$ are indicated in Figs. 2 b,c.

The rate of collisions between excited $\mathrm{Fe}^{+}$ions and $\mathrm{H}$ atoms, with a maximum density of $10^{10} \mathrm{~cm}^{-3}$, is less than one per second. The presence of forbidden lines from the longlived metastable states (lifetime of about $1 \mathrm{~s}$ ) verifies that the relaxation of the excited $\mathrm{Fe}^{+}$ions is purely radiative (Fig. 3). Most important are the "closed" radiative pathways including Ly $\alpha$ pumping, spontaneous decays and stimulated emission, since they provide a collision-free and pure radiative conversion of intense $\operatorname{Ly} \alpha$ radiation into the intense and anomalous $\lambda \lambda 2507 / 09$ UV lines of Fe II (Johansson \& Letokhov 2003).

\section{Spectral peculiarities in Fe II}

Two properties, one atomic and one astrophysical, make the spectrum of Fe II very dominant in spectra of cosmic sources. A complex atomic structure with a large density of energy levels and the high cosmic iron abundance result in numerous lines in ultraviolet and optical spectra - absorption lines in stellar atmospheres and emission lines in nebular regions. Although Fe II has been thoroughly studied in the laboratory and more than 1000 energy levels have been found, ultraviolet stellar spectra still contain unidentified lines of Fe II.

The specific atomic astrophysics problem discussed in the present paper involves two special features that are extremely rare in simple spectra but characteristic for complex spectra: accidental level mixing (ALM) and the presence of "pseudo-metastable" $(P M)$ states. Level mixing is a wellknown effect, which in its simplest form depends on deviations from a pure coupling scheme for the electrons, and a final state is described as a linear combination of, for example, pure $L S$ states. Such mixing of levels may be well predicted by theory even for complex systems. Perturbation and level mixing as a result of configuration interaction are also in general taken care of by detailed calculations. However, two energy levels having the same parity and $J$-value may also mix if they accidentally fall very close to each other in energy. They can have completely different $L S$ character and belong to different

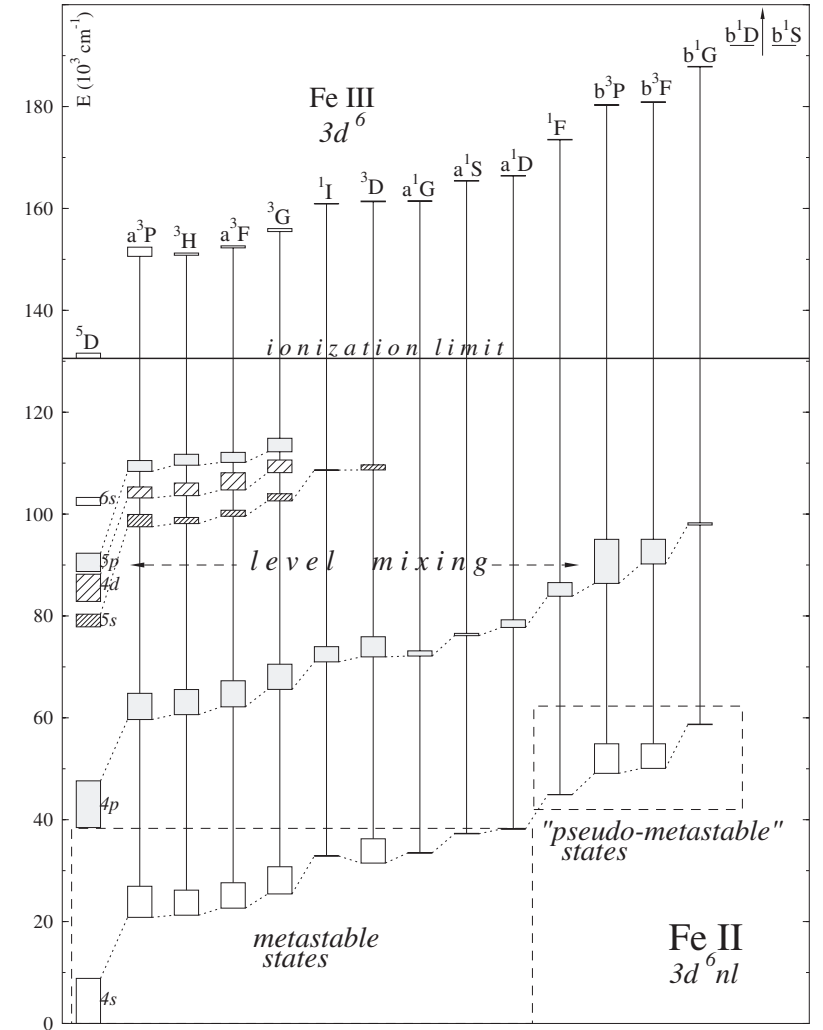

Fig. 4. Partial energy level diagram showing some of the $3 \mathrm{~d}^{6} n l$ configurations of Fe II, where $n l=4 \mathrm{~s}, 4 \mathrm{p}, 5 \mathrm{~s}, 4 \mathrm{~d}$, and $5 \mathrm{p}$. Each configuration is represented by a number of boxes connected by a thin, dotted line. One box contains all (between 2 and 37) energy levels obtained when adding one electron $(n l)$ to one of the $3 \mathrm{~d}^{6}$ parent terms in Fe III. The $4 \mathrm{p}$ and $5 \mathrm{p}$ are odd-parity configurations; the others are of even parity. The lowest dashed box includes the 62 metastable levels, and the smaller box 12 pseudo-metastable levels (see text, Sect. 3). We also mark the level mixing occurring between levels in the left-most $5 \mathrm{p}$ box and two high $4 p$ boxes, all of odd parity.

configurations. Such an $A L M$ is very difficult to predict in quantum mechanical calculations.

The presence of $P M$ states is the crucial feature in the radiative processes discussed in this paper, but before we define the concept "pseudo-metastable" we give a brief description of metastable states. A metastable state in an atom(ion) is a state that cannot decay to a lower state by electric dipole (E1) radiation, i.e. obeying the selection rules for parity and $J$-value. Hence, all states of even parity that are located below the lowest state of odd parity are by definition metastable, and they have radiative lifetimes of the order of $1 \mathrm{~s}$. Metastable states are generally collisionally deexcited but in low-density astrophysical plasmas the probability for collisions is very low. Under such nebular conditions the metastable states decay radiatively in electric quadrupole (E2) or magnetic dipole (M1) transitions observed as forbidden lines. There are 62 metastable states in Fe II belonging to the three configurations $3 d^{6} 4 \mathrm{~s}$, $3 d^{7}$ and $3 d^{5} 4 s^{2}$. One of the three low even-parity configurations containing the metastable states is the ground configuration $3 d^{6} 4$ s. In Fig. 4 we show a condensed energy level diagram for Fe II, arranged according to the "branching rule" for the 

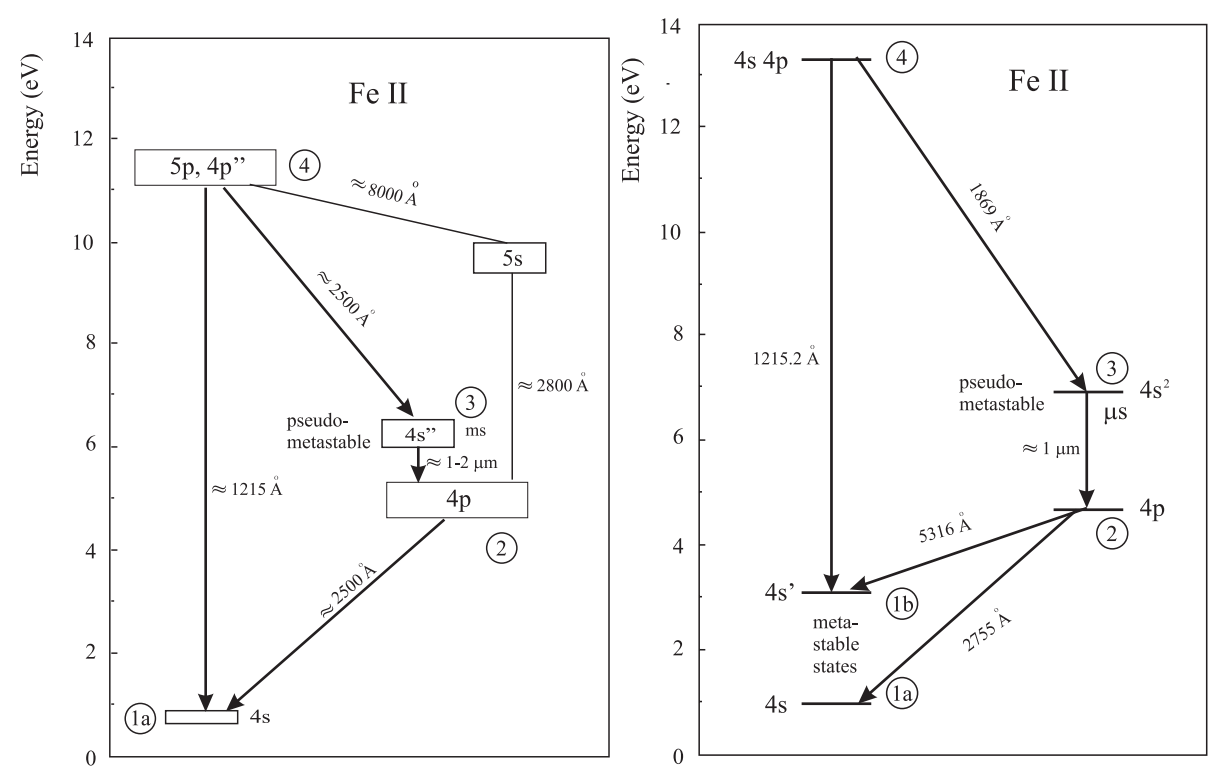

Fig. 5. Schematic energy level diagrams illustrating the three cases of radiative cycles discussed in this paper: a) a real case in Fe II with a combination of the models illustrated in Figs. $2 \mathrm{a}$ and 2b, and b) another Fe II case illustrated in Fig. 2c.

build-up of the structure of complex spectra. Thus, by starting with the next higher ion, Fe III, we add a 4 s-electron to each $L S$-term (the parent term) of the ground configuration $3 \mathrm{~d}^{6}$. The resulting $L S$ terms in Fe II are represented by one box for each parent term. All $4 \mathrm{~s}$ boxes are connected by a dotted line in Fig. 4. Since the $4 \mathrm{~s}$ binding energy is independent of the parent term the pattern of connected $4 \mathrm{~s}$ boxes mimics the pattern of the parent terms. The next higher configuration in the diagram is the odd-parity $4 p$ configuration, represented by grey boxes. Thus, according to the definition all levels in the boxes located below the lowest grey $4 \mathrm{p}$ box are metastable. These are placed in a marked box in the diagram.

Due to the extended structure of the parent terms the $4 \mathrm{~s}$ configuration extends above $4 p$, i.e. the highest $4 \mathrm{~s}$ boxes are located above the lowest $4 p$ box (see Fig. 4). This is a special feature of the transition elements. In Fe II six observed (and two unknown) $L S$-terms of the $3 \mathrm{~d}^{6} 4 \mathrm{~s}$ configuration are located above the lowest $L S$ terms of $3 \mathrm{~d}^{6} 4 \mathrm{p}$, which means that a $4 \mathrm{~s}$ electron can make a downward transition to a $4 \mathrm{p}$ state. The lifetime of these high $4 \mathrm{~s}$ states is of the order of $1 \mathrm{~ms}$, three orders of magnitude shorter than for pure, metastable states but six orders of magnitude longer than for ordinary, excited states. Because of their long lifetimes we call them $P M$ states, and they are marked in a dashed box in Fig. 4. It looks like the PM states keep a fraction of the metastability of their sister LS terms in the same configuration, even if $L S$-allowed decay channels become available. Without looking at the spectroscopic origin of these $P M$ states, but just their $L S$ assignment, it is not possible to distinguish them from other $L S$ terms. However, the transition probability of their decay to $4 p$ is six orders of magnitude smaller than for normal allowed transitions. It turns out that this is the crucial feature behind the explanation of the laser action in Fe II observed in $\eta$ Car and discussed in detail in this paper.

The two cases of four-level schemes in Fe II involved in the radiative processes discussed in the forthcoming sections of this paper are shown in Figs. 5a and 5b. The low 4s box (at $1 \mathrm{eV}$ ) in Fig. $5 \mathrm{a}$ is the $\mathrm{a}^{4} \mathrm{D}$ term in the lowest $4 \mathrm{~s}$ box in Fig. 4. It can be pumped by Ly $\alpha$ to several $5 p$ levels around $11 \mathrm{eV}$, which is discussed in detail in Sect. 3. Due to a near coincidence in energy there is an $A L M$ between some of the lowest $5 p$ levels and some high $4 p$ levels, as indicated in Fig. 4, and a mixing of states from two consecutive members ( $4 p$ and $5 p$ ) in a Rydberg series is very rare. The $A L M$ means that the high $4 p$ levels (denoted $4 \mathrm{p}^{\prime \prime}$ in Fig. $5 \mathrm{a}$ ), which belong to $L S$ terms of the $\left(\mathrm{b}^{3} \mathrm{~F}\right) 4 \mathrm{p}$ and $\left(b^{3} \mathrm{P}\right) 4 \mathrm{p}$ subconfigurations, are also radiatively populated by $\operatorname{Ly} \alpha$. The fast decay from $5 p$ is to $5 s$ (in the near-IR) and back to $4 \mathrm{~s}$ via $4 \mathrm{p}$. The fast decay from $4 \mathrm{p}^{\prime \prime}$ is down to $4 \mathrm{~s}^{\prime \prime}$ in Fig. 5a, i.e. along the vertical line down to $\left(\mathrm{b}^{3} \mathrm{~F}\right) 4 \mathrm{~s}$ and $\left(\mathrm{b}^{3} \mathrm{P}\right) 4 \mathrm{~s}$ in Fig. 4. As all other strong $4 s-4 p$ transitions the corresponding lines fall around $2500 \AA$. These $4 \mathrm{~s}^{\prime \prime}$ levels are the $P M$ states discussed above, and they can decay in slow transitions to the low $4 p$ states at $5 \mathrm{eV}\left(40000 \mathrm{~cm}^{-1}\right)$. The $4 p$ levels have fast decays back to the metastable $4 \mathrm{~s}$ levels, as shown in Fig. 5 a.

The case outlined by the scheme in Fig. 5b starts from a higher metastable $4 \mathrm{~s}$ level (marked $4 \mathrm{~s}^{\prime}$ ), which is excited up to a $4 \mathrm{~s} 4 \mathrm{p}$ state by $\operatorname{Ly} \alpha$ radiation. The natural decay for this pumped state is down to a $4 \mathrm{~s}^{2}$ state at about $7 \mathrm{eV}$. This level belongs to the $3 \mathrm{~d}^{5} 4 \mathrm{~s}^{2}$ configuration, which is not included in Fig. 4. It has about the same properties as the $P M$ states discussed in the first case above, but the lifetime is about three orders of magnitude smaller, or about $1 \mu \mathrm{s}$. The $4 \mathrm{~s}^{2}$ state decays in a "two-electron jump" to $4 p$, i.e. to the same $4 p$ levels as for the $4 \mathrm{~s}^{\prime \prime}$ state in the first case. The $4 \mathrm{p}$ level has a branch to the metastable $4 \mathrm{~s}^{\prime}$ level and even in this case we can find a closed cycle for the radiative excitation and deexcitation.

\section{Population inversion and amplification coefficient in the "bottle-neck" $3 \rightarrow 2$ transition}

There are a number of decay schemes in Fe II creating an inverted population due to a radiative "bottle neck". The main 
schemes are shown in Figs. 5a,b, where few selected energy levels and radiative transitions from the level- and line-rich spectrum of the complex system of Fe II illustrate a photoselective excitation by Ly $\alpha$ and a subsequent cascade of radiative decay channels. A remarkable feature in the decay schemes is the existence of $P M$ states. They decay relatively slowly (radiative lifetimes from $10 \mu \mathrm{s}$ to $1 \mathrm{~ms}$ ) in permitted transitions to lower, rapidly decaying states (lifetimes of a few ns). However, the time elapsing between successive collisions between excited $\mathrm{Fe}^{+}$ions and $\mathrm{H}$ atoms, the main component of the GC with a maximum density of $N_{\mathrm{H}}=10^{10} \mathrm{~cm}^{-3}$, is one second or even longer. Therefore, the relaxation of the Ly $\alpha$ excited $\mathrm{Fe}^{+}$ions occurs without collisions in a purely radiative way.

The cascade of radiative decay of the $\operatorname{Ly} \alpha$ excited states is characterized by a peculiar time hierarchy as illustrated in Fig. 3. On the time scale given in the figure one can distinguish between three relaxation regions: a fast spontaneous decay, a slow spontaneous decay, and a much slower collisional relaxation. Therefore, the spontaneous radiative decay from the Ly $\alpha$ pumped states results inevitably in an inverted population in the transition from the $P M$ state (the $3 \rightarrow 2$ transition in Fig. 5), which acts as a "bottle-neck" for radiative decay in the absence of collisions. If the inverted population density and the optical path length for this transition are large enough, the spontaneous radiation will be amplified due to stimulated emission in the atomic ensemble. The stimulated emission enhances the intensity of the weak lines generated by the slow spontaneous radiative decay of the $P M$ states, and it becomes comparable with the intensity of the fast, spontaneous transitions populating them.

A steady state population inversion is thus achieved in the $3 \rightarrow 2$ transition with a density of

$\Delta N \approx N_{3}-N_{2} \approx N_{3}$,

since $N_{2} \ll N_{3}$ due to the much faster decay of level 2 . The small influence of the statistical weights has been neglected as their ratio is close to one. Since the short-lived levels 4 and 2 can be regarded as empty relative to the long-lived levels 1 and 3 the radiative excitation $W_{\mathrm{exc}}^{14}$ produces the following steady-state distribution of the populations $N_{1}$ (in the initial level 1) and $N_{3}$ (in the long-lived excited level 3):

$N_{3}=N_{1} W_{\mathrm{exc}}^{14} \tau_{3}$,

where $A_{43} \gg W_{\mathrm{exc}}^{14}$. Since the rate of stimulated emission in the $3 \rightarrow 2$ transition, $W_{32}$, is not included in Eq. (3), all Fe II ions can be accumulated in level 3 if $W_{\text {exc }}^{14} \gg 1 / \tau_{3}\left(N_{3} \gg N_{1}\right)$. In the lasing volume, however, $W_{32}$ can be much higher than $1 / \tau_{3}$, and a fast closed radiative cycle will therefore maintain the population of level 1 in such a way that

$N_{3}=N_{1} \frac{W_{\mathrm{exc}}^{14}}{W_{\mathrm{exc}}^{14}+\tau_{3}^{-1}}$,

or even

$N_{3}=N_{1}$,

when the excitation rate is large $\left(W_{\mathrm{exc}}^{14} \gg 1 / \tau_{3}\right)$. We restrict ourselves to make an estimation of the initial amplification without including stimulated transitions (lasing), which will be the subject of future work.

The linear amplification coefficient for the $3 \rightarrow 2$ transition is defined by the standard expression

$\alpha_{32}=\sigma_{32} \Delta N$,

where $\Delta N$ is the inverse population density defined by Eq. (2). The stimulated emission cross section $\sigma_{32}$ is given by

$\sigma_{32}=\frac{\lambda_{32}^{2}}{2 \pi} \frac{A_{32}}{2 \pi \Delta v_{D}}$,

where $\Delta v_{D}$ is the Doppler width of the $3 \rightarrow 2$ transition. At a temperature of $T \approx 100-1000 \mathrm{~K}$ in the relatively cold $\mathrm{HI}$ region, $\Delta v_{D} \approx(300-1000) \mathrm{MHz}$, i.e., $\sigma_{32}=(0.6-2) \times 10^{-13} \mathrm{~cm}^{2}$. Thus, the amplification coefficient may be estimated by the expression

$\alpha_{32}=\sigma_{32}\left(W_{\mathrm{exc}}^{14} \tau_{3}\right) \beta N_{\mathrm{Fe}}$,

where $\beta$ is the fraction of the $\mathrm{Fe}^{+}$ions in state 1 relative to all $\mathrm{Fe}^{+}$ions (all iron atoms in the $\mathrm{HI}$ region of the blob are supposed to be ionized).

The fraction $\beta$ is governed by the excitation rate of level 1 and its radiative lifetime $\tau_{1}$. The excitation rates for collisional population of level $l$ (i.e. by recombination of Fe III and electron collisions) are negligible in comparison with the radiative decay rate $1 / \tau_{1}$ because of the low electron density $\left(n_{\mathrm{e}} \cong\right.$ $\left.10^{4}-10^{5} \mathrm{~cm}^{-3}\right)$ in the HI region. The electrons are generated in the photoionization process of iron as well as other elements having an ionization potential of IP $<13.6 \mathrm{eV}$. However, the most important channel for populating the metastable state 1 is the radiative decay of the high Fe II states selectively excited by Ly $\alpha$ or other intense lines. These excitation channels can provide for an excitation rate $>1 / \tau_{1} \approx 1 \mathrm{~s}^{-1}$ and hence sustain the relative population of state 1 at a level of $\beta \approx 10^{-2}$. This would correspond to an approximately equal distribution of the $\mathrm{Fe}^{+}$ions among the 60 metastable states, including state 1 . Leaving the calculation of a more accurate value of $\beta$ for future modeling, we adopt here a qualitative estimate of $\beta \approx 10^{-2}$. An evidence for a substantial population in the metastable states is provided by the observation of strong, forbidden lines, e.g. from state 1 , in the optical region of $H S T$ spectra of the blob (Zethson 2001).

With these assumptions, we can use Eqs. (1) and (6) to estimate the amplification coefficient for the $3 \rightarrow 2$ transition to $\alpha_{32} \approx\left(3 \times 10^{-18}-10^{-16}\right) N_{\mathrm{Fe}}\left(\mathrm{cm}^{-1}\right)$, where $N_{\mathrm{Fe}^{+}} \approx 10^{-4} \times$ $N_{\mathrm{H}}$. To create a cold HI region in the blob the hydrogen density $N_{\mathrm{H}}$ must exceed a critical value, the so-called critical density $N_{\text {cr }} \approx 10^{8} \mathrm{~cm}^{-3}$ (Johansson \& Letokhov 2001a,b). Thus, for $\alpha_{32} \geq\left(3 \times 10^{-14}-10^{-12}\right) \mathrm{cm}^{-1}$ and a blob diameter of $D \approx$ $10^{15} \mathrm{~cm}$, which can be regarded as the size $L$ of the amplifying region, $\alpha L \approx(30-100)$ at an effective blob temperature of Ly $\alpha$ of $T_{\alpha} \approx(12-18) \times 10^{3} \mathrm{~K}$. The amplification coefficient $\alpha_{32}$ is, according to Eq. (7), sensitive to the spectral width of the amplified line. From this point of view the cold HI region is more suitable as a lasing medium than the hotter HII region, causing a higher Doppler width in the $3 \rightarrow 2$ transition.

Let us emphasize that the amplification coefficient $\alpha_{32}$ according to Eqs. (7)-(8) does not depend strongly on the 
Einstein coefficient $A_{32}$, since $A_{32} \sim 1 / \tau_{3}$ as long as the branching fraction for the $3 \rightarrow 2$ transition is dominating in the decay of state 3 . The $(\alpha L)$-values obtained correspond to fairly high values of the linear (non-saturated) amplification coefficient $K=$ $\exp (\alpha L)$. In the saturation regime, however, the intensity of the weak line $\lambda_{32}$ (in photons $/ \mathrm{cm}^{2} \mathrm{~s}$ ) approaches that of the strong line $\lambda_{43}$. Thereafter, the amplification regime becomes saturated. This means that the rate of stimulated transition approaches the pumping rate of level 3 provided by the spontaneous $4 \rightarrow 3$ decay. Under such conditions the intensities of both these lines grow in proportion to the propagation length $L$.

\section{A radiative millisecond "bottle neck" and strong radiative cycling}

Many of the radiative decay schemes of Ly $\alpha$ pumped Fe II levels contain three spontaneous transitions before the electron ends up in a low-lying metastable state. In some of these schemes there is a high probability that the final state of the electron is the same as the initial state, i.e. there is a high branching fraction of the final transition connecting states 2 and 1 . This is the case illustrated in Fig. 5a.

First we discuss the closed cycle containing $\operatorname{Ly} \alpha$ absorption, spontaneous decay with emission of the bright UV $\lambda \lambda 2507 / 2509$ lines, stimulated emission at 1.68 and $1.74 \mu \mathrm{m}$, and a fast UV transition to the initial state. The diagram in Fig. 6 includes a few of the known energy levels of Fe II, which together with the inserted transitions and atomic data are relevant in the present study of a strong radiative cycle. The broad (a few hundreds $\mathrm{cm}^{-1}$ ) Ly $\alpha$ line almost coincides in wavelength with two Fe II transitions from the low level $\mathrm{a}^{4} \mathrm{D}_{7 / 2}$ (level 1 in Fig. 6) to the close levels $\left({ }^{5} \mathrm{D}\right) 5 \mathrm{p}{ }^{6} \mathrm{~F}_{9 / 2}^{0}$ and $\left(\mathrm{b}^{3} \mathrm{~F}\right) 4 \mathrm{p}{ }^{4} \mathrm{G}_{9 / 2}^{0}$ (marked 4 in Fig. 6), the frequency difference (detuning) being $\Delta v=-160 \mathrm{~cm}^{-1}$ and $-185 \mathrm{~cm}^{-1}$, respectively. The detuning is compensated by the Doppler shift or the broadening of $\operatorname{Ly} \alpha$ (see Sect. 8). The line at $2509.1 \AA$ to state $3\left(\mathrm{c}^{4} \mathrm{~F}_{7 / 2}\right)$ is the main decay channel from $\left(b^{3} F\right) 4 p{ }^{4} G_{9 / 2}^{0}$ (state 4 ) with a branching fraction of about $\gamma \approx 0.9$. Due to the small energy difference there is an $A L M$ between the two energy levels in state 4 , $\left({ }^{5} \mathrm{D}\right) 5 \mathrm{p}{ }^{6} \mathrm{~F}_{9 / 2}^{0}$ and $\left(\mathrm{b}^{3} \mathrm{~F}\right) 4 \mathrm{p}^{4} \mathrm{G}_{9 / 2}^{0}$, resulting in similar decay schemes (Johansson 1978). As discussed above level 3 is a $P M$ state, with a lifetime of $1.5 \mathrm{~ms}$, but in contrast to a pure, metastable state it can decay slowly by electric dipole radiation to the short-lived states $2\left(\mathrm{z}^{4} \mathrm{D}_{7 / 2}^{0}\right.$ and $\left.\mathrm{z}^{4} \mathrm{~F}_{9 / 2}^{0}\right)$. To get strong radiative cycling it is important that state 2 decays by returning a large fraction of the $\mathrm{Fe}^{+}$ions to the initial state 1 , which is metastable with a radiative lifetime of about one second. Assuming the blob conditions discussed in the previous section $\left(N_{\mathrm{H}} \approx 10^{9}-10^{10} \mathrm{~cm}^{-3}\right)$ the time scale for collisions is much longer than the time scale for radiative decay of state 3 . Under these conditions the closed radiative cycle $1 \rightarrow 4 \rightarrow 3 \rightarrow 2 \rightarrow 1$ has a "bottle neck" in the $3 \rightarrow 2$ transition.

The linear amplification coefficient for the $3 \rightarrow 2$ transitions at $1.74 \mu \mathrm{m}$ and $1.68 \mu \mathrm{m}$ (Fig. 9) is defined for $W_{\mathrm{exc}}^{14} \ll \tau_{3}^{-1}$

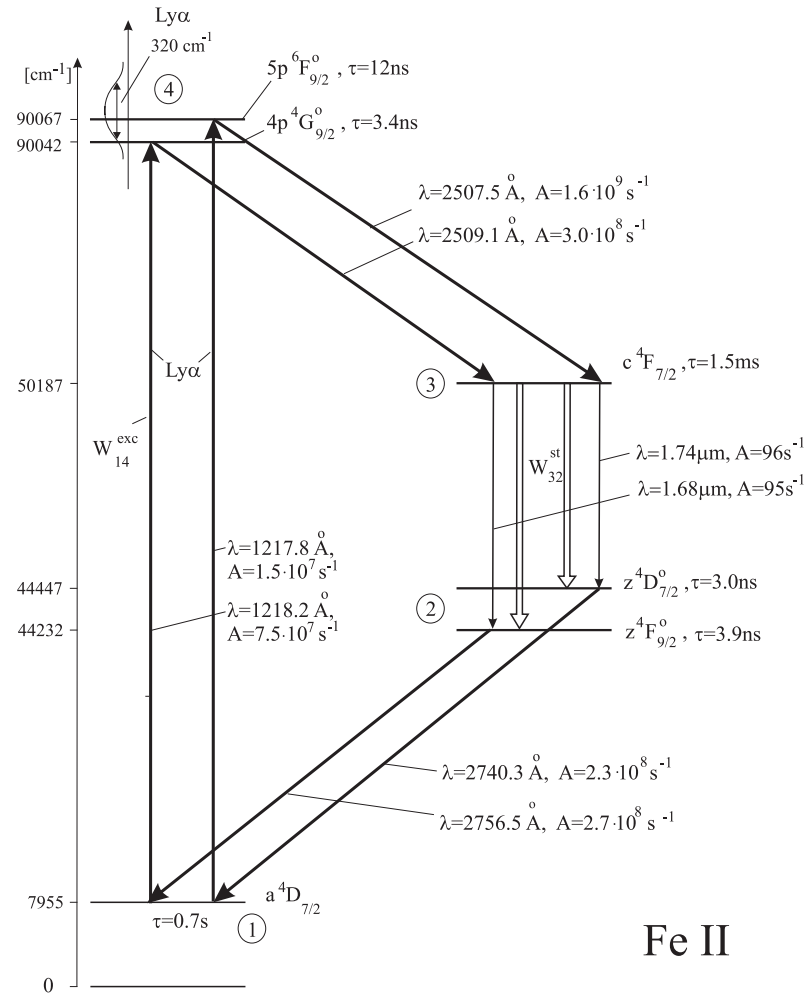

Fig. 6. A partial energy level diagram (with parameter values) of Fe II showing a closed loop with strong radiative cycling. The cycle includes a selective photo-excitation of the highest level 4 by intense $\operatorname{Ly} \alpha$ radiation and a millisecond pseudo-metastable "bottleneck", causing population inversion. (Lifetimes and $A$-values are from Bergeson et al. 1996; Kurucz 2002; Raassen 2002).

(a rather moderate requirement) by Eqs. (4) and (8), rewritten as:

$\alpha_{32}=\left[\frac{\lambda_{32}^{2}}{2 \pi} \frac{A_{32}}{2 \pi \Delta v}\right] \tau_{3}\left(\beta N_{\mathrm{Fe}}\right)$.

From Eq. (9) we get that the amplification coefficient $\alpha_{32}\left(\mathrm{~cm}^{-1}\right)$ for the scheme in Fig. 6 is largely determined by the factor $\left(A_{32} \tau_{3}\right)$. Its maximum value is $A_{32} \tau_{3}=1$ in the ideal case, i.e. when there is only one radiative channel of spontaneous decay from state 3 . In the real case (see Fig. 6) discussed here the factor $A_{32} \tau_{3} \approx 0.15$. Rough estimates of $\alpha_{32} L \geq 10$, where $L \approx 10^{15} \mathrm{~cm}$ is the length of the amplifying medium, can be obtained for $T_{\alpha}>10000 \mathrm{~K}$ and $N_{\mathrm{H}} \simeq 10^{8} \mathrm{~cm}^{-3}$ or $T_{\alpha}>8000 \mathrm{~K}$ and $N_{\mathrm{H}} \simeq 10^{9} \mathrm{~cm}^{-3}$.

For an ensemble of atoms with sufficient density and size the channel $3 \rightarrow 2$ will open for a fast stimulated decay, and the duration of the whole radiative cycle is determined by the rate of the slowest excitation channel rather than by the $W_{\mathrm{exc}}^{14}$ rate of the slowest spontaneous decay $A_{32}$. For a high brightness temperature of Ly $\alpha$ in the range $T_{\alpha} \approx 15000 \mathrm{~K}$ the excitation rate is $W_{\mathrm{exc}}^{14} \approx 5 \times 10^{3} \mathrm{~s}^{-1}$ giving a duration of the total radiative cycle in Fe II of $\tau_{\text {cycle }} \approx 2 \times 10^{-4}$ s. Hence, the Fe ions can undergo a maximum number of cycles $\left(\tau_{1} / \tau_{\text {cycle }} \approx 3.5 \times 10^{3}\right)$ during the lifetime of the initial state $\left(\tau_{1}\right)$.

A large number of radiative cycles containing a stimulated channel provide a large intensity enhancement of the 
$\lambda \lambda 2507 / 2509$ fluorescence lines $(4 \rightarrow 3)$ due to the suppressed accumulation of $\mathrm{Fe}^{+}$ions in the $P M$ state 3 and the corresponding depletion of the initial state 1 . If the density $N_{\mathrm{Fe}}$ and the size $L$ are not sufficiently large for the ensemble of atoms the amplification will be small and the stimulated channel will not operate. In such a case the radiative "bottle neck" limits the rate of the radiative cycling in Fe II to the time $\tau_{1}=1.5 \mathrm{~ms}$ and the intensities of the two UV lines $\lambda_{43}$ become normal.

High-resolution spectra of the blobs outside $\eta$ Car, spatially resolved from the central star, show the anomalous brightness of the two $\lambda \lambda 2507 / 2509$ lines. Further observational evidence in the blob spectrum for the radiative cycle is the presence of one "bottle neck" line at $8674.7 \AA$ (Zethson 2001), which is an intercombination line with an extremely low transition probability $A_{32} \approx 13 \mathrm{~s}^{-1}$ (Raassen 2002). The "bottle neck" transitions around $1.7 \mu \mathrm{m}$ indicated in Fig. 6 have been observed in ground-based spectra of $\eta$ Car (Hamann et al. 1994). The $3 \rightarrow 2$ transition from $\mathrm{c}^{4} \mathrm{~F}_{7 / 2}$ at $\lambda=1.74 \mu \mathrm{m}$ and $1.68 \mu \mathrm{m}$ appear relatively stronger than lines from the other fine structure levels of $\mathrm{c}^{4} \mathrm{~F}$. Since the ground-based spectra of $\eta$ Car contain integrated light from a larger region than the Weigelt blobs discussed here the absolute intensities cannot be compared with the intensities measured in the $H S T$ spectra.

Moreover, according to the observations made in (Gull et al. 2001), the UV lines of the $2 \rightarrow 1$ transition at 2740 and $2756 \AA$ have an integrated intensity comparable with that of the bright lines of the $4 \rightarrow 1$ transition. The width of these spectral lines is great because of the substantial optical density in the $4 \rightarrow 1$ transition and the corresponding resonance transfer radiation broadening. Resonance radiation trapping increases the effective lifetime of level 2, but still it remains much shorter than the long lifetime of level 3 and does not prevent the formation of an inverted population in the $3 \rightarrow 2$ transition.

\section{Radiative microsecond "bottle-neck" and weak radiative cycling.}

In some of the schemes of Ly $\alpha$ pumped Fe II levels there is a low probability that the final state of the electron is the same as the initial state, i.e. there is a low branching fraction of the final transition connecting states 2 and 1 . Such a case is illustrated in Fig. 5b. The primary spontaneous decay of state 2 is to the metastable state $l a$ at $1 \mathrm{eV}$, and a weaker branch populates the initial state ( $l b$ at about $3 \mathrm{eV}$ ) of the closed radiative cycle. In the case studied here state $l a$ is the same as state 1 in the millisecond "bottle-neck" discussed in Sect. 5, which gave a strong radiative cycling. The low branching fraction of $0.1 \%$ for the $2 \rightarrow 1 \mathrm{~b}$ transition at $5116 \AA$ produces a closed cycle but a weak radiative cycling. Some feeding of the initial level, state $l b$, is provided by the radiative cycle discussed in Sect. 5 , which means that there is only need for an initial population in state $1 a$ to start the pumping processes of both the ms- and $\mu \mathrm{s}-$ "bottle-neck" cycles.

A more detailed scheme of the microsecond "bottle-neck" with relevant atomic data is given in Fig. 7. The Ly $\alpha$ radiation populates a high-lying state of Fe II from the metastable state $\mathrm{a}^{4} \mathrm{G}_{11 / 2}$. This excitation scheme is operative in various objects, verified by the observation of the spontaneous radiative

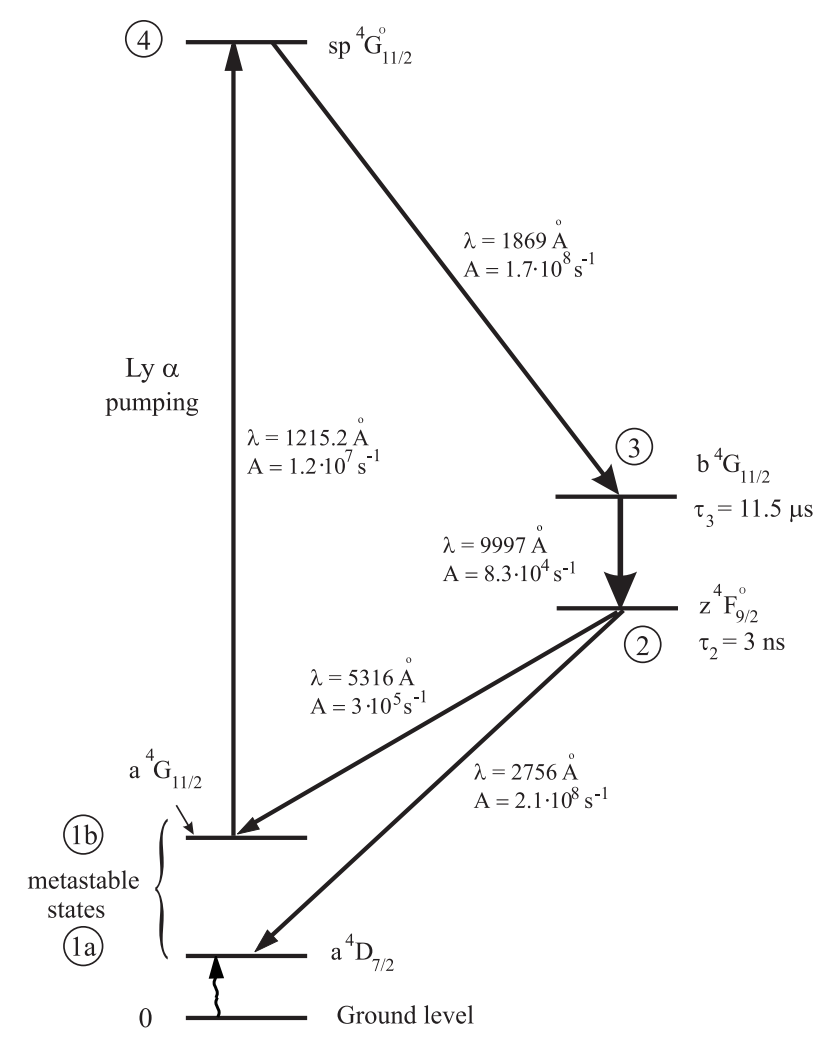

Fig. 7. Diagram showing the creation of population inversion in the $3 \rightarrow 2$ transition of Fe II because of a microsecond "bottle-neck". Since only a small fraction of the $\mathrm{Ly} \alpha$ pumped Fe II ions return to the initial state $1 \mathrm{~b}$ the closed loop $1 \mathrm{~b} \rightarrow 4 \rightarrow 3 \rightarrow 2 \rightarrow 1 \mathrm{~b}$ generates a weak radiative cycling.

decay in the primary cascade as a fluorescence line at $1869 \AA$ (Johansson \& Jordan 1984) as well as the secondary cascade at $9997 \AA$, seen in many objects as a prominent feature. Based on the extended laboratory analysis of Fe II (Johansson 1978), the $9997 \AA$ line was first identified (Johansson 1977) in the near-IR- spectrum of $\eta$ Car (Thackeray 1969). A plausible explanation to its appearance in spectra of some astrophysical objects was ascribed to the excitation scheme shown in Fig. 7 (Johansson 1990).

According to theoretical calculations (Kurucz 2003), the main radiative decay from state $4,3 \mathrm{~d}^{5}\left(\mathrm{a}^{2} \mathrm{~F}\right) 4 \mathrm{~s} 4 \mathrm{p}\left({ }^{3} \mathrm{P}\right)^{4} \mathrm{G}_{11 / 2}^{0}$ (hereafter $\mathrm{sp}^{4} \mathrm{G}$ ), occurs to the fine-structure levels of $\mathrm{b}^{4} \mathrm{G}$ with branching fractions of 0.83 to $J=11 / 2$ and 0.04 to $J=9 / 2$. The most natural representative of state 3 in Figs. $5 \mathrm{~b}$ and 7 is thus $b^{4} \mathrm{G}_{11 / 2}$ with a radiative lifetime of $11 \mu \mathrm{s}$. Hence, it has a relatively slow spontaneous decay, $A_{32}=8 \times 10^{4} \mathrm{~s}^{-1}$, to level 2 $\left(\mathrm{z}^{4} \mathrm{~F}_{9 / 2}^{0}\right)$, which has a much shorter lifetime $(\approx 3 \mathrm{~ns})$.

Using HST/STIS, Gull et al. (2001) have recorded spectra of blob B in $\eta$ Car at high angular and spectral resolution. The spectrum contains the intense $9997 \AA$ line (see Fig. 8), which can be excited by Ly $\alpha$ radiation according to the excitation and decay scheme shown in Fig. 7. The $9997 \AA$ line is thus a transition from a microsecond $P M$ state, which belongs to a different configuration $\left(3 \mathrm{~d}^{5} 4 \mathrm{~s}^{2}\right)$ than the millisecond $P M$ state in Sect. 5. Since the lower state of the $9997 \AA$ transition is short-lived (a few ns) an inverted population is built up, and the 


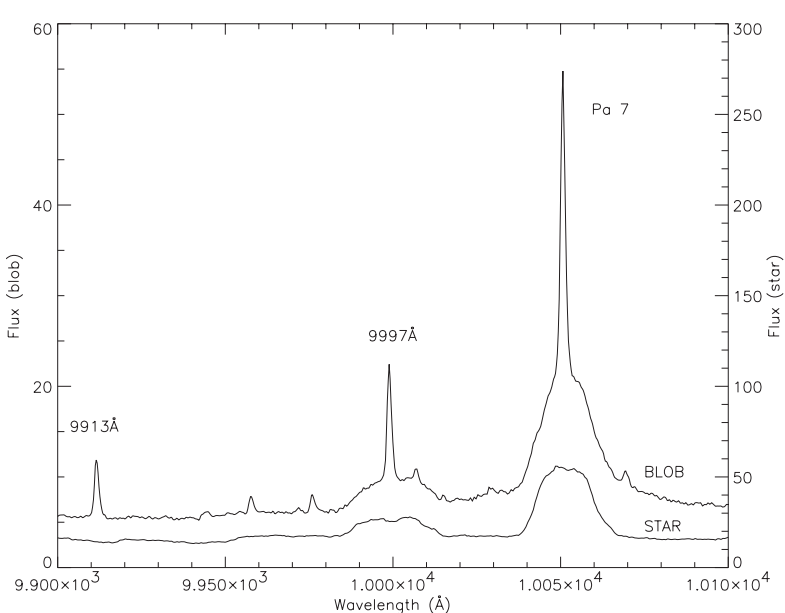

Fig. 8. Comparison of HST/STIS spectra of the central star (flux scale on right axis) and Weigelt blob B (flux scale on left axis) in $\eta$ Car showing intensity enhanced blob lines at 9913 and $9997 \AA$ (see text).

gain is determined by the rate of photoexcitation provided by the Ly $\alpha$ radiation. The high intensity is naturally explained by stimulated emission of radiation, and the amplification coefficient of this transition is about one order of magnitude higher than in the case of the ms "bottle-neck" because of the factor $\left(A_{32} \tau_{3}\right)$ in Eq. (9).

\section{Combination of strong and weak radiative cycles in a millisecond "bottle neck"}

The existence of a great number of metastable states and the high density of high-lying energy levels in FeII cause several coincidences between absorption lines and $\operatorname{Ly} \alpha$ and thereby the generation of inverted population. In this section we give examples of cascade schemes from the same millisecond "bottle neck" but with both strong and weak radiative cycles.

In Fig. 9 we present two transition schemes, which provide for the amplification of the extremely weak FeII lines at $9391.5 \AA, 9617.6 \AA$ and $9913.0 \AA$ and some other lines around $2 \mu \mathrm{m}$. The difference between the two groups is only the $P M$ states, which have different $\mathrm{J}$-values but belong to the same $L S$ term. The two schemes represent a combination of the previous ones discussed in Sects. 6 and 7 due to the fact that there are two sets of possible "bottle neck" $3 \rightarrow 2$ transitions. In one set state 2 consists of sextet levels explaining the extremely low transition probability $A_{32}$ in the intercombination lines at $\lambda \lambda 9391 / 9617$ in Fig. 9a and $\lambda 9913$ in Fig. 9b. Since the sextet levels decay mainly to the ground term in Fe II and only in a very slow decay to the initial level, the closed $1 \rightarrow 4 \rightarrow 3 \rightarrow 2 \rightarrow 1$ radiative cycle becomes very weak. However, like the case studied in Sect. 6 the $P M$ states can also decay to quartet levels in state 2 , which combine strongly with the initial state. The corresponding $P M$ transitions occur in the near-IR region around $2 \mu \mathrm{m}$.

The amplification coefficient for the $2 \mu \mathrm{m}$ lines should be of the same order of magnitude as for the $1.7 \mu \mathrm{m}$ lines in Fig. 6 in Sect. 5, whereas it is reduced for the lines in the range 9300-9900 A due to the smaller $A$ value. The reduction of the Einstein coefficients $A_{32}$ has, in principle, no effect on the amplification coefficient, provided that the upper level 3 decays radiatively only to one low laser level. However, this is not the case here, and the faster decay to the quartet levels in a time of $\tau_{3} \approx A_{32}^{-1}$ reduces the amplification coefficient (6) for the transitions to the sextet levels by a factor of $A_{32} \tau_{3} \approx 10^{-2}$. But, the great gain margin is sufficient to ensure $\alpha L \geq 10$, even for the weak lines, and all three lines mentioned above are observed and identified in the blob spectrum of $\eta$ Car (Zethson 2001). Two of them, the laser lines at $9617 \AA$ and $9913 \AA$, are shown in Fig. 10 as intense features in the blob spectrum but not observed in the stellar spectrum. Moreover, the two lines at $9617.6 \AA$ and $9391.5 \AA$ should be very weak in spontaneous emission and have an intensity ratio of 2.5 , determined by the branching ratio (the ratio of the $A$-values). These lines, however, are observed (Gull et al. 2001) as intense lines with equal intensities. This is naturally explained by their formation through a stimulated mechanism, which is operative under saturated excitation conditions with a common pumping source and a common upper level. The "peculiar" intensity ratio observed for the $\lambda \lambda 9391 / 9617$ lines forms perhaps the strongest argument for lasing (Hillier, private communication). The laser lines around $2 \mu \mathrm{m}$ in the strong radiative cycle of Figs. 9a,b appear, like the $1.7 \mu \mathrm{m}$ lines in Fig. 6, as prominent features in the ground spectrum of $\eta$ Car (Hamann et al. 1994). The absence of the laser lines in the stellar spectrum (see Fig. 10) is due to collisional relaxation of the $P M$ states in the stellar atmosphere/wind implying a Boltzmann population distribution.

\section{Sources of Ly $\alpha$ Radiation Pumping Fe II in the Weigelt blobs}

The blobs in $\eta$ Car are embedded in the photospheric radiation and the wind from the central star. Let us speculate about possible sources for Ly $\alpha$ radiation capable of pumping Fe II within the blobs. Firstly, the Ly $\alpha$ radiation emitted by the photosphere of $\eta$ Car can excite $\mathrm{Fe}^{+}$ions in blobs located at distances of $R_{\mathrm{b}} \approx(300-1000) \cdot r_{\mathrm{s}}$, where $r_{\mathrm{s}} \approx 10^{13} \mathrm{~cm}$ is the stellar radius. But, even at such a relatively close location of the blob the dilution factor $\Omega=\left(r_{\mathrm{s}} / 2 R_{\mathrm{b}}\right)^{2}=10^{-6}-10^{-5}$, which substantially weakens the intensity of the Ly $\alpha$ radiation reaching the blob and makes it incapable of producing the bright 2507/2509 $\AA$ lines (Johansson \& Letokhov 2001).

The stellar wind of $\eta$ Car is an important characteristic of the star's surroundings, the mass loss rate of the star being substantial (Davidson \& Humphreys 1997). Spherical (Hillier et al. 2001) as well as aspherical (Smith et al. 2003) models of the stellar wind of $\eta$ Car with a terminal velocity of $v_{1} \simeq 500-1000 \mathrm{~km} \mathrm{~s}^{-1}$ have been considered. In accordance with models by Lamers \& Cassinelli (1999) of the stellar wind its velocity is almost close to the terminal value everywhere between $\eta \mathrm{Car}$ and the GC. The Lyman continuum radiation, $\mathrm{Ly}_{\mathrm{c}}$, from the star photoionizes the stellar wind and after the subsequent recombination a wide Ly $\alpha$ profile is produced. Another potential source of ionizing radiation is a binary companion, but in the discussion below about $\operatorname{Ly} \alpha$ formation inside the blob it is not important to distinguish between the individual components in a binary system. It is possible to consider three cases of irradiation of the blob by radiation from the stellar 

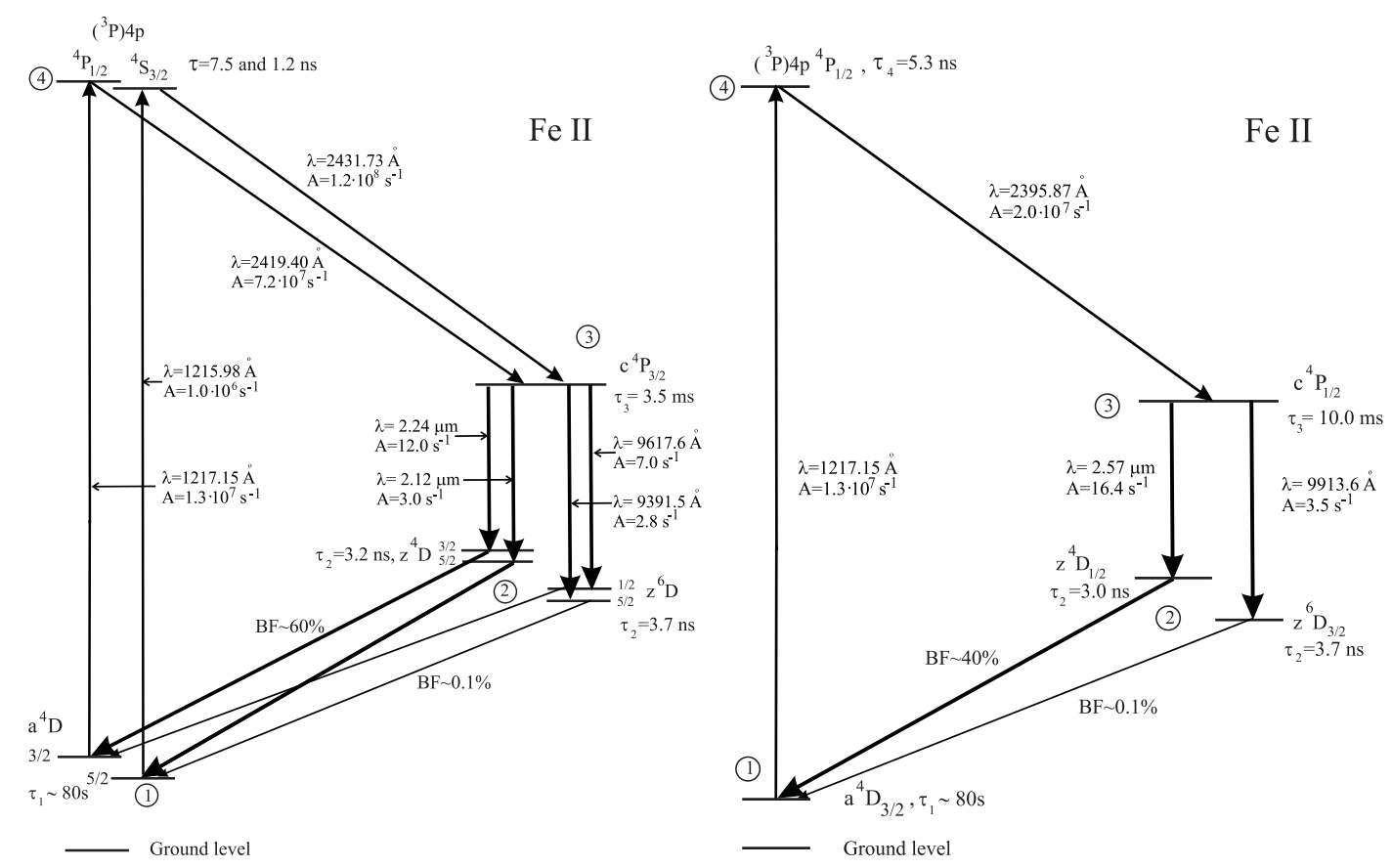

Fig. 9. Partial energy level diagrams of Fe II showing two examples of combined strong and weak radiative cycling due to alternative decay routes for the two "pseudo-metastable" states $c^{4} \mathrm{P}_{3 / 2}$ (Fig. 9a) and $\mathrm{c}^{4} \mathrm{P}_{1 / 2}$ (Fig. 9b). The "bottle-neck" effect generates inverted population and lasing in the $3 \rightarrow 2$ transitions to sextet and quartet levels, which have quite different branching fractions (BF) to the initial state 1 .

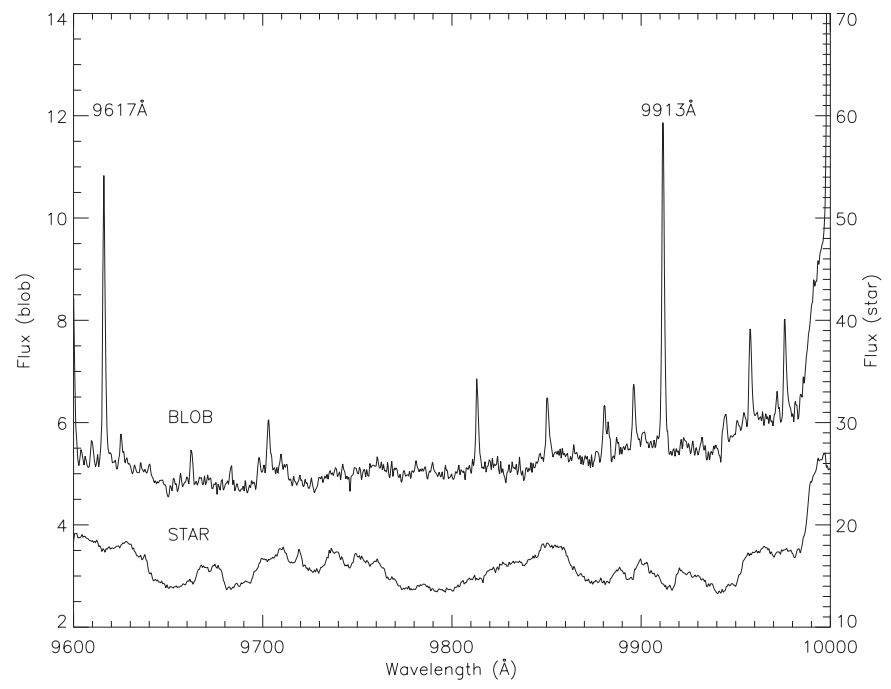

Fig. 10. Comparison of HST/STIS spectra of the central star (flux scale on right axis) and the Weigelt blob B (flux scale on left axis) in $\eta$ Car with the two lasing lines $\lambda \lambda 9617,9913$ generated in the level schemes in Fig. 9 and discussed in Sect. 7.

wind depending on the size of its Strömgren radius, $\tilde{R}_{\text {Str }}$ relative to the distance $R_{\mathrm{b}}$ between the blob and the star as illustrated in Figs. 11a-c.

1) $\tilde{R}_{\mathrm{Str}} \gg R_{\mathrm{b}}$ (Fig. 11a). In this case the $\mathrm{Ly}_{\mathrm{c}}$ radiation reaches the blob without significant attenuation and can photoionize the front part of it. If the hydrogen density in the blob exceeds the critical density $N_{\mathrm{cr}} \simeq 10^{8} \mathrm{~cm}^{-3}$ (Klimov et al. 2002) the Strömgren boundary of the blob, defined by, intersects the blob. The front part of the blob produces an intense Ly $\alpha$ radiation with $T_{\text {alpha }}>10000 \mathrm{~K}$. Thus, the dilution of the photospheric Ly $\alpha$ radiation falling on to the blob is to a great degree compensated by spectral compression of absorbed broadband radiation to a $10^{3}-10^{4}$ times more narrow Ly $\alpha$ profile (Johansson \& Letokhov 2004). Under these conditions the blob is very different from a typical planetary nebula since the dilution factor $\Omega=\left(r_{\mathrm{s}} / 2 L\right)^{2}$ is many orders of magnitude greater than for a planetary nebula $\left(\Omega \simeq 10^{-15}-10^{-12}\right)$.

2) $\tilde{R}_{\text {Str }} \ll R_{\mathrm{b}}$ (Fig. 11b). In this case the Lyman continuum is blocked out for the blob. This means that the entire volume of space between $\eta$ Car and the blob is an ionized H II region, with the Strömgren boundary for the stellar wind being located between the star and the blob. Since hydrogen is not fully ionized the blob does not have two regions as in the previous case, where the HII and HI zones are adjacent to each other (Fig. 11a). A single-component blob (a HI region) is probably too cold to emit the bright UV Fe II lines. It will therefore make sense to assume that the optical density of the Lyman continuum $\tau\left(\lambda_{\mathrm{c}}\right) \leq 1$ in the region between $\eta$ Car and the blob.

3) $\tilde{R}_{\text {Str }} \geq R_{\mathrm{b}}+D$ (Fig. 11c). In this case the Strömgren boundary outside the blob is located slightly behind it. This can be considered as an optimal situation for pumping of Fe II in the blob for two reasons. Firstly, the Ly $\alpha$ photons produced in this torus-like zone beyond the blob are red-shifted and $\Delta \lambda_{w}=\lambda_{0}\left(v_{t} / c\right) \cos \Theta$, where $\Delta \lambda_{w}=$ the wavelength shift, $\lambda_{0}=$ the rest wavelength, $v_{t}=$ the terminal stellar wind velocity and $\Theta$ is the viewing angle as indicated in Fig. 11c. The red-shifted and broadband Ly $\alpha$ radiation irradiates the $\mathrm{H} I$ region of the blob without any need for diffusion Doppler broadening. It can penetrate the whole H I region, since its optical density $\tau\left(\lambda_{0}+\Delta \lambda\right)$ in the far 
a)

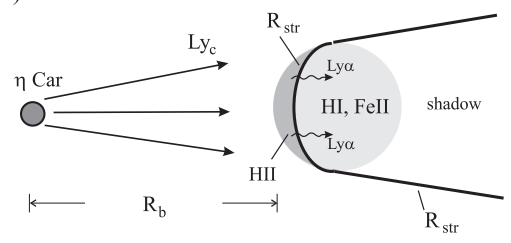

b)
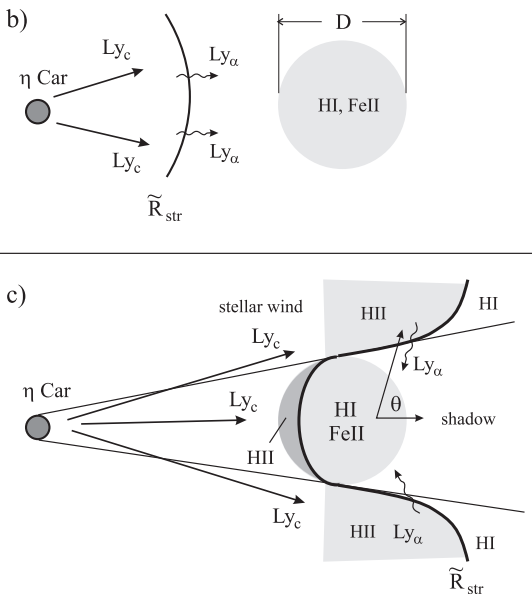

Fig. 11. A schematic model of a Weigelt blob, located at a distance $R_{\mathrm{b}}$ from the hot, luminous star $\eta$ Car and subject to the stellar radiation and wind. a) A local Strömgren boundary, $R_{\text {str }}$, between the $\mathrm{H}$ II and $\mathrm{H}$ I regions is located inside the blob. Stellar radiation in the Lyman continuum $\left(\mathrm{Ly}_{\mathrm{c}}, \mathrm{h} v>\mathrm{h} v_{\mathrm{c}}=13.6 \mathrm{eV}\right)$ photo-ionizes hydrogen only in the front part of the blob. Radiation in the range $7.6 \mathrm{eV}<h v<13.6 \mathrm{eV}$ passes through the $\mathrm{H}$ II region and photo- ionizes iron atoms, which have a density of $N_{\mathrm{Fe}} \simeq 10^{-4} N_{\mathrm{H}}$ in the $\mathrm{H}$ I region. Intense Ly $\alpha$ radiation from the $\mathrm{H}$ II region excites Fe II resonantly in the $\mathrm{H}$ I region (lasing volume). The Strömgren boundary for the stellar wind $\tilde{R}_{\text {str }} \gg R_{\mathrm{b}}$ b) the stellar wind is optically thick for $\mathrm{Ly}_{\mathrm{c}}$ and the blob is a one-component H I medium. Only remote, blue- shifted Ly $\alpha$ radiation from the stellar wind can irradiate the HI region containing $\mathrm{Fe}$ II; c) the Strömgren boundary in the wind lies behind the blob $\left(\tilde{R}_{\text {str }} \approx R_{\mathrm{b}}\right.$ ), providing intense, red-shifted Ly $\alpha$ photons for efficient excitation of Fe II.

wing of $\operatorname{Ly} \alpha$ is not abnormally high, where $\Delta \lambda=2.4 \AA$ is the shift of the Fe II absorption line relative to the center of $\operatorname{Ly} \alpha$.

Secondly, the dilution factor for the Lyman continuum irradiating the stellar wind (see geometry in Fig. 11c) will largely be compensated for by the conversion of $\mathrm{Ly}_{\mathrm{c}}$ into narrower $\mathrm{Ly} \alpha$ radiation in the stellar wind in the vicinity of the blob. The optimal case is when the size of $\tilde{R}_{\text {Str }}$ coincides with the distance to the irradiated part of the HI region in the blob, which will require a significant density of the stellar wind near the blob. The critical hydrogen density in the stellar wind, $\tilde{N}_{\mathrm{cr}}$, that can provide $\tilde{R}_{\mathrm{Str}} \simeq R_{\mathrm{b}}$, is connected with the critical density of the blob itself, $N_{\mathrm{cr}}$, required to locate the Strömgren boundary inside the blob, by the approximate expression:

$\tilde{N}_{\mathrm{cr}} / N_{\mathrm{cr}} \simeq\left(D / R_{\mathrm{b}}\right)^{1 / 2}$.

For the adopted values of the distance to the star $R_{\mathrm{b}} \simeq$ $300-1000 r_{\mathrm{s}} \simeq 10^{16}-3 \times 10^{16} \mathrm{~cm}$ and the size of the blob,
$D \simeq 10^{15} \mathrm{~cm}, \tilde{N}_{\mathrm{cr}}$ is some tenths of $N_{\mathrm{cr}} \simeq 10^{8} \mathrm{~cm}^{-3}$, i.e. a relatively high density of the stellar wind close to the blob. This very approximate estimate of $\tilde{N}_{\text {cr }}$ in the vicinity of the blob $\left(0.15^{\prime \prime}\right.$ from $\eta$ Car) agrees with other estimates, based on observed and modelled spectra of the stellar wind (Hillier 2001, 2003). The evaluation of Eq. (10) agrees with direct measurements of the size and shape of the stellar wind and the blobs in $\eta$ Car (van Boekel et al. 2003). Of course, all these qualitative speculations should be subject of detailed modelling of the stellar wind with the blob included, but that is out of the scope of the present paper.

In Sects. 4 and 5 above we have used the effective spectral temperature of $\operatorname{Ly} \alpha\left(T_{\alpha}>10000 \mathrm{~K}\right)$, in the treatment of the pumping of absorption lines to achieve a population inversion in some Fe II transitions from $P M$ states. This requirement is valid for any possible source of intense Ly $\alpha$ radiation. The consideration of possible Ly $\alpha$ pumping from the stellar wind is still preliminary and qualitative, and it needs a future detailed modelling.

\section{On the spectral brightness and spectral width of astronomical lasers}

We have emphasized that the radiation intensity (photons $\mathrm{s}^{-1} \mathrm{~cm}^{-2}$ ) of an astrophysical laser (APL) line cannot be higher than the intensity of the pumping line. In principle the spectral brightness (photons $\mathrm{s}^{-1} \mathrm{~cm}^{-2} \mathrm{~Hz}^{-1} \mathrm{sr}^{-1}$ ) of the APL line can exceed that of the pumping line because of two effects: 1) the narrowing of the APL line by resonant amplification of the radiation (both the spontaneous radiation itself and some external radiation); and 2) an anisotropic type of amplification (for example in an elongated amplifying region). Since these effects are interrelated, they can be considered simultaneously.

In the regime of linear amplification of spontaneous emission, the line width decreases because of an exponential amplification at the line center. This is described by a simple relation (Casperson \& Yariv 1972; Allen \& Peters 1972):

$\Delta v=\frac{\Delta v_{D}}{\sqrt{1+\alpha L}}$

where $\alpha$ is the gain coefficient (see Sect. 4) per unit length. The narrowing occurs until the amplified line, which is inhomogeneously Doppler-broadened, gets saturated and its peak "flattens out". This effect was considered for the APL-saturated amplifier, and it has been shown (Litvak 1970) that the narrowing of the spectrum lines ceases at saturation. The line broadens again to the width of the gain line, which has been confirmed by observational data for astrophysical masers (Reid \& Moran 1981; Elitzur 1992).

It would be very interesting to measure the sub-Doppler line width of the $9997 \AA$ line (as well as other laser lines mentioned above) using a spectral resolution $R>10^{6}$ and an angular resolution better than 0.1 arcsec. This could, for example, be done with spatially separate telescopes using a heterodyne correlation analysis of the Brown-Twiss effect (Johnson et al. 1974). The spectral brightness of APL radiation can grow as a result of the narrowing of the spectrum lines. In order to observe this effect, one will need a detection technique that is 
capable of very high spectral resolution. Such methods are known in radio-frequency spectroscopy, but are as yet unavailable in the optical region. However, such measurements in the optical region using Brown-Twiss-Townes heterodyne interferometry are in principle quite feasible (Lavrinovich \& Letokhov 1976; Letokhov 1996). An analysis of performing such a direct proof of the laser effect in the Weigelt blobs will be discussed elsewhere (Johansson \& Letokhov, in preparation).

\section{Conclusion: On the difference of the evidence of astrophysical lasers and masers}

Space lasers with optical pumping operate according to a scheme similar to the one suggested for the first laboratory laser 45 years ago by A. Schawlow and C. Townes (1958). We believe that laser amplification and stimulated emission of radiation is a fairly common and widespread phenomenon, at least for gaseous condensations in the vicinity of bright stars. This is due to the occurrence of two types of processes (fast radiative and slow collisional) in the very rarefied gaseous condensations, whereby the population of energy levels in atoms (ions) can relax. These relaxation processes occur on highly different time scales, radiative relaxation operating on a wide time scale of $10^{-9}-10^{-3} \mathrm{~s}$ (sometimes even within $10^{-3}-1 \mathrm{~s}$ ), and collisional relaxation, on a time scale of over seconds (at gas densities $<10^{10} \mathrm{~cm}^{-3}$ ). In the case of photoselective excitation of some high-lying electronic levels of an atom or ion with a complex energy-level structure, radiative relaxation can take place as a consequence of downward transitions with spontaneous emission of radiation, in the course of which there develops an inverse population of some pair (pairs) of levels. If the size of a gas cloud is large enough, large amplification on the inverted-population transition automatically switches on the radiative relaxation channel, which leads to faster stimulated transitions until collisional relaxation becomes important. Thus, the laser action is an intrinsic characteristic of the radiative cooling of gas clouds near bright stars by stimulated emission for inverted transitions along with spontaneous emission for normal transitions.

However, the detection of the laser effect is less evident in the optical region of the spectrum than in the microwave region, where it gives rise to strong radiation lines of exceptionally high brightness temperature $\left(10^{10}-10^{15} \mathrm{~K}\right.$ (Elitzur 1992)). In the optical region, the laser effect raises the intensity of weak forbidden lines up to the intensity of those strong allowed lines providing the selective photoexcitation of the upper levels. This is explained by the huge difference $\left(\approx 10^{15}-10^{18}\right.$ times) between the spontaneous emission rates and inverse population production mechanisms in the two wavelength regions. This follows from simple qualitative considerations of the steadystate saturation regime of the isotropic maser/laser action.

The stimulated emission of radiation in a space maser occurs as a result of pumping of the upper maser level, which is not associated with any spontaneous emission in radiative microwave transitions. It may therefore have a decay rate much in excess of the spontaneous emission rate, which lies in the region $A_{\mathrm{mn}} \simeq 10^{-9}-10^{-7} \mathrm{~s}^{-1}$. As a result, the intensity of the stimulated radiation can be many orders of magnitude higher than the intensity of the spontaneous radiation and it is only limited by the pumping rate. This is exactly the reason why the brightness temperature of maser microwave lines reaches as high a value as $10^{10}-10^{15} \mathrm{~K}$. The intensity of maser lines is not borrowed from other microwave spectral lines, which are very weak, but from other pumping sources.

In the optical region of the spectrum, the rate of allowed spontaneous transitions is high $\left(A_{\mathrm{mn}} \simeq 10^{8}-10^{9} \mathrm{~s}^{-1}\right)$, and it is precisely spontaneous transitions that provide for the pumping of the upper level in an optical space laser at a sufficiently high rate to exceed the rates of collisional pumping mechanisms. This is especially true for the case considered here where the space laser is being indirectly pumped by HLy $\alpha$ in the vicinity of $\eta$ Car, one of the most luminous stars of our Galaxy. Therefore, the intensity of the stimulated radiation in the optical region generated by the occurrence of an inversion population and significant amplification cannot in the steady-state regime to any substantial extent exceed the intensity of the pumping spectral lines, formed by spontaneous emission in allowed radiative transitions of atoms or ions. This fact presents difficulties in detecting laser action by a large enhancement of the intensity of the radiation, but it should manifest itself in comparatively moderate changes of the branching ratios of spectral lines, having a common source of pumping.

One exception is the case of quantum transitions having a relatively low spontaneous emission probability $\left(A_{\mathrm{mn}} \simeq \mathrm{a}\right.$ few $\left.10^{5} \mathrm{~s}^{-1}\right)$, and consequently the spontaneous radiation lines are weak. Once an inverted level population has developed in such a transition with a significant amplification in a properly sized cloud, a stimulated emission channel opens up. The stimulated transition rate cannot be much higher than the spontaneous emission rate, which is limited by the pumping rate of the upper laser level. Thus, the intensity of the laser line should become comparable with the intensity of the lines, which are due to allowed spontaneous emission of radiation and resulting from the optical pumping (direct or indirect) of the upper level. This takes place only in the inverted population volume with size $L \gg 1 / \alpha$, where $\alpha$ is the amplification coefficient per unit length. In such a case, a spectral line, expected to be weak, must appear as a spectral line of normal intensity, typical for an allowed transition. This is exactly what we have found to be the case with several spectral lines of Fe II pumped indirectly by the intense H Ly $\alpha$ radiation (1215 $\AA$ ) in the Weigelt blobs of $\eta$ Car.

In conclusion, let us emphasize that stimulated emission at optical and near-infrared wavelengths forming non-collisional, radiative cycling of atomic particles in astrophysical media gives a natural explanation to the anomalous behaviour of spectral lines of two types: i) the transformation of a very weak line from a PM state have a low transition probability (line $3 \rightarrow 2$ in Figs. $1 a, 6$ and 9) into a strong line with an intensity comparable to that of an allowed transition; and ii) the transformation of a normally intense line into an anomalously bright line (line $4 \rightarrow 3$ in Fig. 10) due to radiative cycling, which is only possible if stimulated emission drives the weakest link in the cycle.

Before astrophysical spectra at very high resolution can be observed these conclusive facts constitute, at present, the only 
proofs of the Fe II astrophysical laser in the Weigelt blobs of $\eta$ Carinae.

Acknowledgements. We thank Dr. John Hillier for sending us data on the density of the stellar wind, and later for providing very useful referee comments on the manuscript. V.S.L. acknowledges financial support through grants (S.J.) from the Royal Swedish Academy of Sciences and the Wenner-Gren Foundations, as well as Lund Observatory for hospitality and the Russian Foundation for Basic Research (grant No. 103-02- 16377). The research project is supported by a grant (S.J.) from the Swedish National Space Board.

\section{References}

Allen, L., \& Peters, G. I. 1972, Nature, 235, 143

Bergeson, S. D., Mullman, K. L., Wickliffe, M. E., et al. 1996, ApJ, 464, 1044

Betz, A. L., McLaren, R. A., Sutton, E. C., \& Townes, C. H. 1976, ApJ, 208, L145

van Boekel, R., Kervella, P., Schöller, et al. 2003, A\&A, 410, L37

Bowen, I. 1935, ApJ, 81, 1

Brown, S., Jordan, C., \& Wilson, R. 1979, In The First Year of IUE, ed. A. Willis, 232, UCL

Casperson, L. W., \& Yariv, A. 1972, IEEE J. Quant. Electron., 8, 80

Cheung, A. C., Rank, D. M., Townes, C. H., Thornton, O. D., \& Wehl, W. J. 1969, Nature, 221, 626

Davidson, K., \& Humphreys, R. M. 1997, ARA\&A, 35, 1

Elitzur, M. 1992, Astronomical Masers (Dordrecht: Kluwer Academ. Publ.)

Gull, T. R., Ishibashi, K., Davidson K., \& the Cycle 7 STIS GO Team 1999, in Eta Carinae at the Millenium, ed. J. A. Morse, R. M. Humphreys, \& A. Damineli, ASP Conf. Ser., 179, 144

Gull, T. R., Ishibashi, K., Davidson, K., \& Collins, N. 2001, in Eta Carinae and Other Mysterious Stars, ed. T. Gull, S. Johansson, \& K. Davidson, ASP Conf. Ser., 242, 391

Hamann, F., De Poy, D. L., Johansson, S., \& Elias, J. 1994, ApJ, 422, 626

Harper, G. M., Wilkinson, E., Brown, A., Jordan, C., \& Linsky, J. L. 2001, ApJ, 551, 486

Hartman, H., \& Johansson, S. 2000, A\&A, 359, 627

Hillier, J. D., Davidson, K., Ishibashi, K., \& Gull, T. 2001, A\&A, 553, 837

Hillier, J. D. 2003, private communication

Johansson, S. 1978, Phys. Scri., 18, 217

Johansson, S. 1990, in Cools Stars, Stellar Systems, and the Sun, ed. G. Wallerstein, ASP Conf. Ser., 9, 307

Johansson, S., \& Jordan, C. 1984, MNRAS, 210, 239
Johansson, S., \& Letokhov, V. 2001a, in Eta Carinae and Other Mysterious Stars: The Hidden Opportunities of Emission Line Spectroscopy, ASP Conf. Ser., 242, 309

Johansson, S., \& Letokhov, V. 2001b, A\&A, 378, 266

Johansson, S., \& Letokhov V. 2002, JETP Lett., 75(10), 495

Johansson, S., \& Letokhov V. 2003, Phys. Rev. Lett., 90(1), 01110-1

Johansson, S., \& Letokhov, V. 2004, Astron. Lett., 30(1), 67

Johansson, S., Zethson, T., Hartman, H., \& Letokhov, V. 2001, in Eta Carinae and Other Mysterious Stars, ed. T. Gull, S. Johansson, \& K. Davidson, ASP Conf. Ser., 242, 297

Johnson, M. A., Betz, A. L., \& Townes, C. H. 1974, Phys. Rev. Lett., 33,1617

Jordan, C. 1998, J. Opt. Soc. Amer., 5, 2252

Jordan, C. 1998, in Physics of Formation of Fe II Lines Outside LTE, ed. R. Viotti, A. Vittone, \& M. Friedjung (Dordrecht: D. Reidel Publ. Co.)

Klimov, V., Johansson, S., \& Letokhov, V. 2002, A\&A, 385, 311

Kimble, R. A., Woodgate, B. E., Bowers, C. W., et al. 1998, ApJ, 492, L83

Kurucz, R. L. 2002, http://cfaku5 . harvard.edu/atoms .html

Lamers, H., \& Cassinelli, J. 1999, Introduction to Stellar Wind (Cambridge, UK: Cambridge Univ. Press)

Lavrinovich, N. N., \& Letokhov, V. S. 1974, Sov. Phys. - JETP, 40, 800

Letokhov, V. S. 1972, IEEE J. Quant. Electron. QE- 8, 615

Letokhov, V. S. 1996, in Amazing Light. A Volume Dedicated to Charles Hard Townes on His 80th Birthday, ed. R. Y. Chiao (Berlin: Springer), 409

Litvak, M. M. 1970, Phys. Rev. A, 2, 2107

Mumma, M. J., Buhl, D., Chin, G., et al. 1981, Science, 212, 45

Netzer, H. 1998, in Physics of Formation of Fe II Lines Outside LTE, ed. R. Viotti, A. Vittone, \& M. Friedjung (Dordrecht: D. Reidel Publ. Co.), 247

Raassen, A. J. J. 2002, http://www. science. uva.nl/pub/orth/iron

Reid, M. J., \& Moran, J. M. 1981, ARA\&A, 19, 231

Schawlow, A. L., \& Townes, C. H. 1958, Phys. Rev., 112, 1940

Smith, N., Davidson, K., Gull, T. R., Ishibashi, K., \& Hillier, J. D. 2003, ApJ, 586, 432

Strelnitskii, V., Haas, M. R., Smith, H. A., et al. 1996, Science, 272, 1459

Thackeray, A. D. 1969, Mon. Not. Astr. Soc. South. Afr., 28, 37

Townes, C. H. 1997, Quant. Elect., 27, 1031

Verner, E. M., Gull, T. R., Bruhweiler, F., et al. 2002, ApJ, 581, 1154

Weaver, H., William, D. R. W., Dietler, N. H., \& Lum, W. E. 1965, Nature, 208, 29

Weigelt, G., \& Ebersberger, J. 1986, A\&A, 163, L5

Zethson, T. 2001, Ph.D. Thesis Lund University 